\title{
Method of analysis and monitoring of the electromechanical converters parameters based on a linear integral criterion using sensitivity models
}

\author{
N. A. Malev ${ }^{1, *}$, A. I. Mukhametshin ${ }^{1}, O . V$. Pogoditsky $^{1}$, and W. M. Mwaku ${ }^{2}$ \\ ${ }^{1}$ Kazan State Power Engineering University, Kazan, Russia \\ ${ }^{2}$ Copperbelt University, Kitwe, Zambia
}

\begin{abstract}
During the electromechanical converters operation in the composition of the working sets, objects parameters can change both under the influence of external factors, such as changes in the environmental characteristics, and due to parametric disturbances caused by changes of the physical characteristics of electromechanical converters elements. In this regard, the analysis and monitoring of electromechanical converters parameters is an important task. The article deals with a method that allows to ensure control over the operation of electromechanical converters during operation as part of working sets, based on variations analysis of the object parameters. The article provides a linear integral criterion computational scheme using the reference and sensitivity models to the parameters to be analyzed. The results provide a good way to estimate changes of the electromechanical converters parameters with the required accuracy.
\end{abstract}

\section{Introduction}

The electromechanical converters performance in the composition of working sets in various industries is determined by dynamics and accuracy in the steady state. These characteristics should be as invariant as possible to changes of the electromechanical converters parameters. Parameters variations can be driven by external forces (operating conditions) which, in turn, leads to the product wear and tear, as well as features of the object operation, leading to parametric disturbances.

External disturbances, constituting interferences, caused by the force fields influence external to the researched object are referred to as coordinate or additive $[1,2,3,4,5,6]$. Considering the abovementioned there is a following general formula of electromechanical converter motion equation:

$$
\mathbf{A} \dot{\mathbf{y}}(t)=\mathbf{B x}(t)+\mathbf{C f}(t),
$$

where $\dot{\mathbf{y}}(t), \mathbf{x}(t), \mathbf{f}(t)$ - vectors of output and input signals and external influences, respectively; A, B, C coefficient matrices

Parametric disturbances are driven by changes of physical characteristics of the object elements and lead to the following form of the differential motion equation (1):

$$
(\mathbf{A}+\Delta \mathbf{A}) \dot{\mathbf{y}}(t)=(\mathbf{B}+\Delta \mathbf{B}) \mathbf{x}(t)+(\mathbf{C}+\Delta \mathbf{C}) \mathbf{f}(t)
$$

From (1) and (2) it follows that the coordinate and parametric disturbances have a fundamental difference in the action it produces to the electromechanical converter: coordinate disturbances affect only the forced motion, while parametric affect both the forced and free motion components, that is, parametric disturbances affect both the transient and steady-state processes $[7,8,9]$. In this regard, modernization of analyzing and monitoring methods of the electromechanical transducers parametric disturbances is essential both in theoretical studies at the design phase and in engineering practice during day-today operation [10].

\section{Methods}

For the electromechanical converters performance monitoring, it is advisable to use a refined mathematical model of the object, gathered from the studies using an experimental assembly. DC motor type 4PB112M2G was designated as targets for research. Specification is presented in Table $1[11]$.

Based on the abovementioned specification, the following motor parameters were calculated:

- armature winding active resistance

\footnotetext{
*Corresponding author: maleeev@ mail.ru
} 
Table 1. DC motor 4PB112M2G specification.

\begin{tabular}{|c|c|c|c|c|c|c|c|}
\hline $\begin{array}{c}\text { Armature } \\
\text { voltage } \\
U_{a}, \mathrm{~V}\end{array}$ & $\begin{array}{c}\text { Power } \\
P, \mathrm{~kW}\end{array}$ & $\begin{array}{c}\text { Armature } \\
\text { current } \\
I_{a}, \mathrm{~A}\end{array}$ & $\begin{array}{c}\text { Efficiency } \\
\eta, \%\end{array}$ & $\begin{array}{c}\text { Rotational } \\
\text { speed } \\
n, \mathrm{rpm}\end{array}$ & $\begin{array}{c}\text { Number of } \\
\text { pole pairs, } \\
2 p\end{array}$ & $\begin{array}{c}\text { Maximum rota- } \\
\text { tional speed } \\
n_{\max }, \mathrm{rpm}\end{array}$ & $\begin{array}{c}\text { Moment of } \\
\text { inertia } \\
J, \mathrm{~kg} \cdot \mathrm{m}^{2}\end{array}$ \\
\hline 220 & 3 & 16.2 & 80 & 3000 & 2 & 4000 & 0.0235 \\
\hline
\end{tabular}

$R_{a}=0.5(1-\eta) \frac{U_{a}}{I_{a}}=0.5(1-0.8) \frac{220}{16.2}=1.358 \mathrm{Ohm}$

- armature winding inductance [12]

$L_{a}=k_{i} \frac{30 U_{a}}{\pi p I_{a} n}=0.5 \frac{30 \cdot 220}{\pi \cdot 2 \cdot 16.2 \cdot 3000}=0.0108 \mathrm{H}$

- back EMF constant

$c=\frac{U_{a}-I_{a} R_{a}}{\omega}=\frac{220-16.2 \cdot 1.358}{314}=0.63 \mathrm{~V} \cdot \mathrm{s} / \mathrm{rad}$

On the basis of conducted calculations, DC motor transfer function is obtained:

$$
W(s)=\frac{\Omega(s)}{U_{a}(s)}=\frac{1 / c}{\frac{L_{a} J}{c^{2}} s^{2}+\frac{R_{a} J}{c^{2}} s+1}
$$

The study was conducted by means of an experimental assembly with DC motor powered by fourquadrant thyristor converter type SPRINT ELECTRIC 3600XRi. Rotational speed information comes from the tachogenerator installed on the motor shaft. The signal transmitted from the tachogenerator arrives at voltage divider with a 60: 1 transfer ratio and then at the eightchannel serial analog-digital converter NI 4472 input with a sampling frequency of $20 \mathrm{kHz}$. The ADC connects to the PCI bus of the personal computer.

\section{Results}

As a result of the experiment, time-response characteristics were obtained corresponding to a mathematical model of a second-order DC motor (6) taking into consideration a thyristor converter with the following parameters values of its transfer function: transfer ratio $K_{u z}=22$; time constant $T_{u z}=0.00173 \mathrm{~s}$.

Further, a detailed study of the DC motor was carried out in order to comparative evaluation of the dependencies of the rotational speed experimentally obtained $\Omega_{d c . e}(t)$ using the Simulink-model of the object $\Omega_{d c . m}(t)$. figure 1 .

Transient curves $\Omega_{d c . e}(t)$ and $\Omega_{d c . m}(t)$ can be seen in

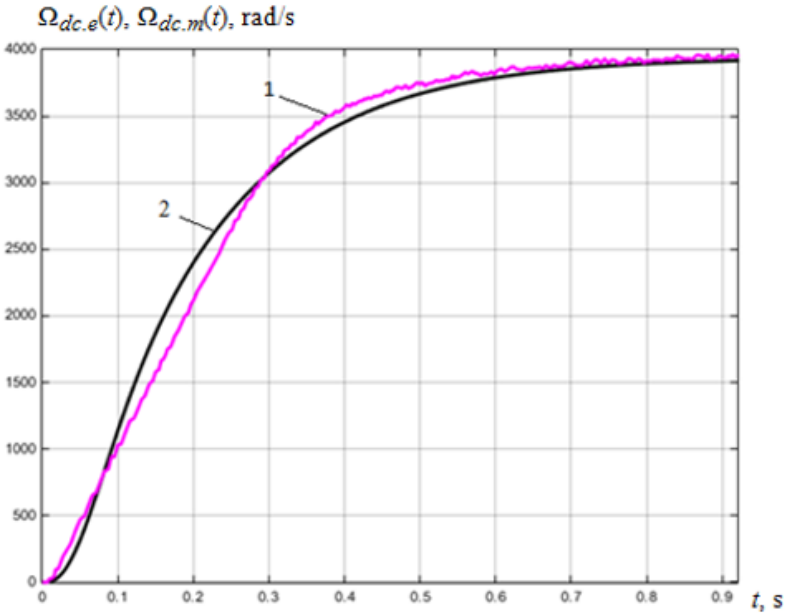

Fig. 1. Transient curves: $1-\Omega_{d c . e}(t) ; 2-\Omega_{d c . m}(t)$.

As can be seen from figure 1, transients continue for $0.92 \mathrm{~s}$, which corresponds to the time of the experiment. Upon completion of the experiment the rotational speed values $\Omega_{d c . e}^{s e t}(t)=3950 \mathrm{rad} / \mathrm{s}, \Omega_{d c . m}^{s e t}(t)=3920 \mathrm{rad} / \mathrm{s}$, while the relative error does not exceed $0.76 \%$ in the steady state.

To produce a scheme for analyzing and monitoring the DC motor parameters, a searchless gradient method was used with reference and sensitivity models [13, 14, $15,16,17,18]$ for the parameters to be analyzed in order to obtain a discriminant $\varepsilon$ proportional to the parameters variations.

The method involves the functional $I$ minimization from discrepancy $\varepsilon$ by calculating the gradient by variable parameters $\chi=\left\{\chi_{1}, \chi_{2}, \ldots, \chi_{n}\right\}$. In the accepted functional $I(\varepsilon)=\varepsilon^{2}[19,20]$, the components of the gradient of the functional are determined by partial derivatives:

$$
\frac{\partial I(\varepsilon)}{\partial \chi_{i}}=2 \varepsilon \frac{\partial \varepsilon}{\partial \chi_{i}}
$$

The gradient-based algorithm for estimating the parameters $\chi_{i}$ from the condition of attaining the minimum of the functional $I(\varepsilon)$ can be written in scalar form as:

$$
s \chi_{i}=-\lambda_{i} \varepsilon \frac{\partial \varepsilon}{\partial \chi_{i}}
$$

Next we have 


$$
\begin{aligned}
& \frac{\partial \varepsilon}{\partial \chi_{i}}=\frac{\partial}{\partial \chi_{i}}\left(\omega_{o i}-\omega_{m i}\right)= \\
& =-\frac{\partial \omega_{m i}}{\partial \chi_{i}}=-\frac{\partial W_{m}(s, \chi) u}{\partial \chi_{i}}=W_{\chi}(s, \chi) u,
\end{aligned}
$$

where $W_{\chi}(s, \chi)=-\frac{\partial W_{m}(s, \chi)}{\partial \chi_{i}}-$ sensitivity function.

A structural scheme of the analysis and monitoring of the parameters of the study object using the reference and sensitivity models and the linear integral criterion [21] calculation is presented in figure 2 .

The following notation is agreed in the scheme: $W_{o}(s, \chi)$-study object model; $\chi$ - object parameter vector; $W_{m}(s, \chi) \quad-$ object reference model; $\boldsymbol{\Lambda}_{\chi}=\left\{\lambda_{1}, \lambda_{2}, \ldots, \lambda_{n}\right\} \quad-$ vector of numerical coefficients; $\frac{\partial W_{m}(s, \chi)}{\partial \chi_{i}}-$ sensitivity model by parameters analyzed; $Q(\chi)$ - linear integral criterion (integral estimate); $f[Q(\chi)]$ - functional dependence (static response); $k_{i}=\frac{\chi_{o i}}{\chi_{m i}}-$ relative change of the i-th component of the object parameter vector.

In accordance with the structural scheme of parameters analysis and monitoring presented in Figure 2, an intermediate Simulink-model was obtained, the output of which is a linear integral criterion for each of

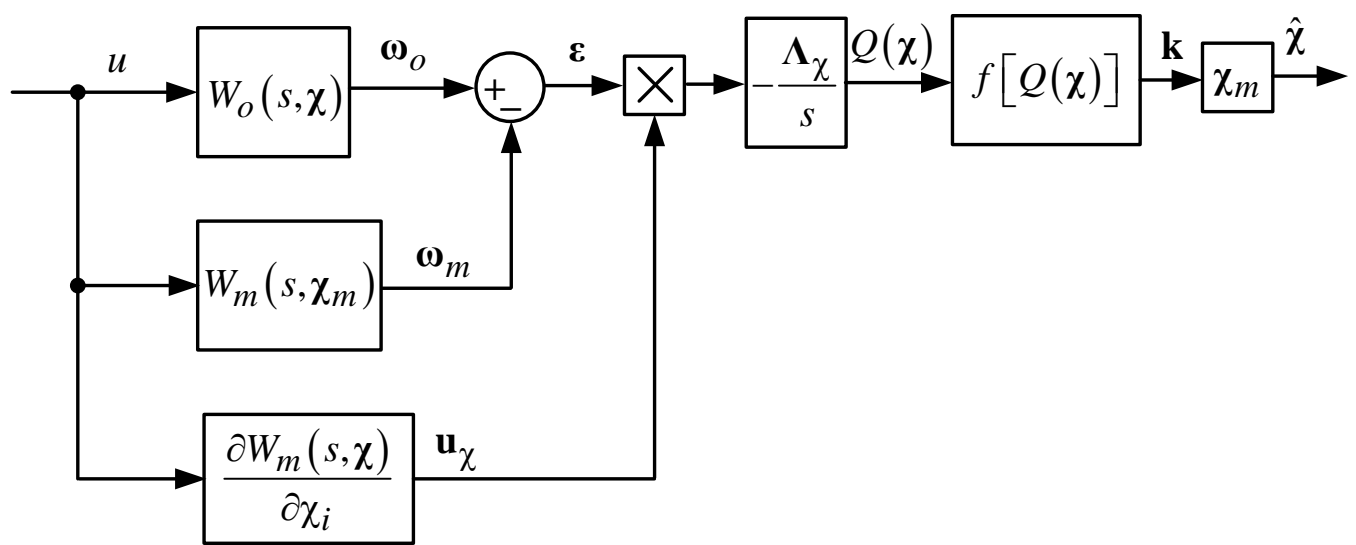

Figure 2. Structural scheme of parameters analysis and monitoring.

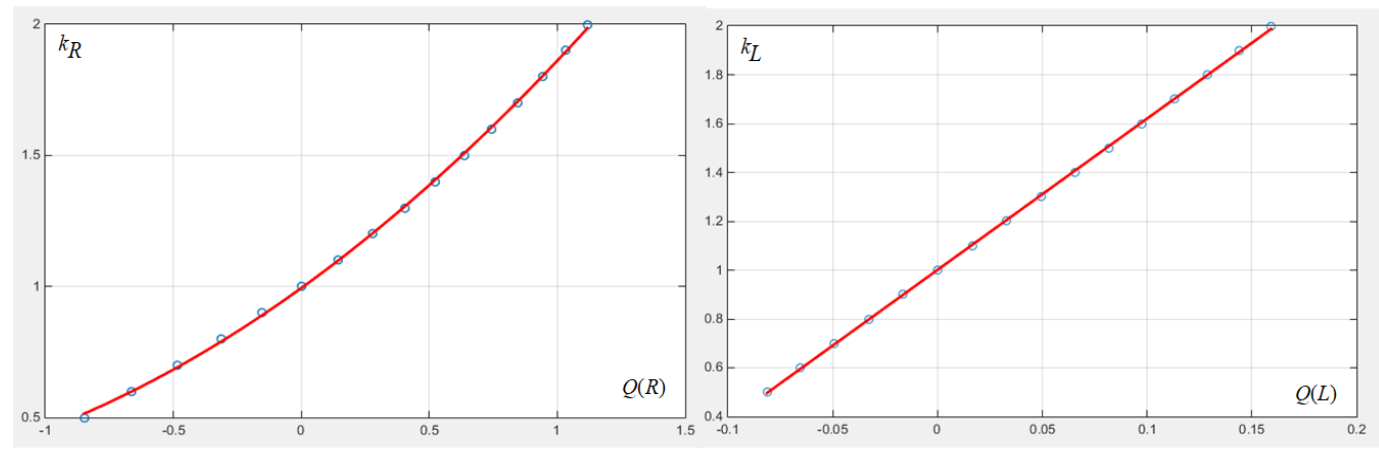

Figure 3. Functional dependencies: a) $f[Q(R)]$; b) $f[Q(L)]$.

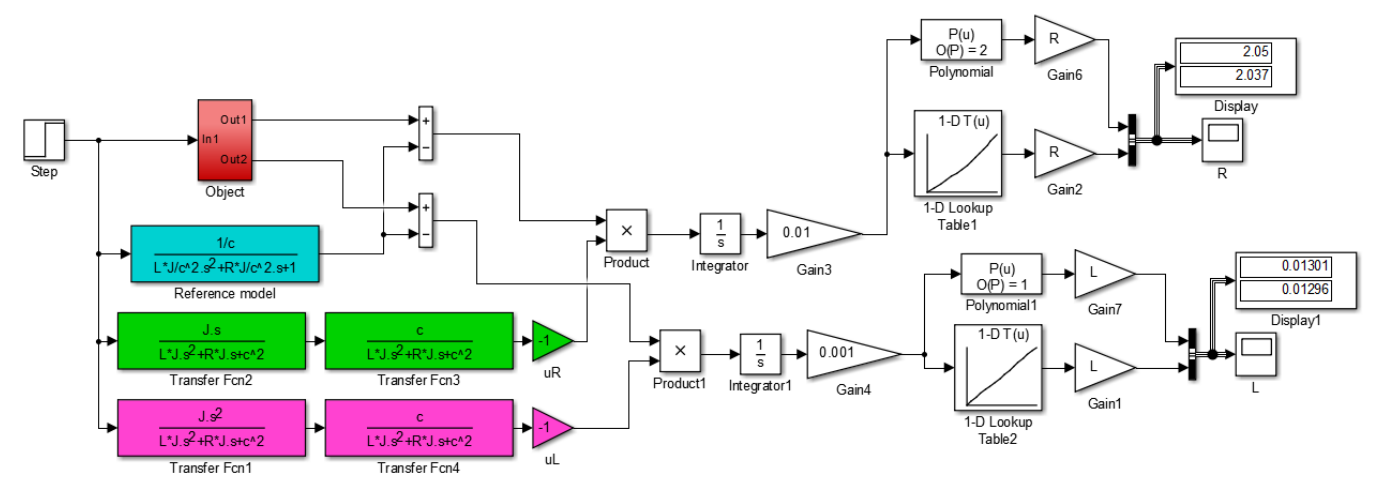

Figure 4. Simulink-model of the analysis and control of the active resistance and armature inductance. 


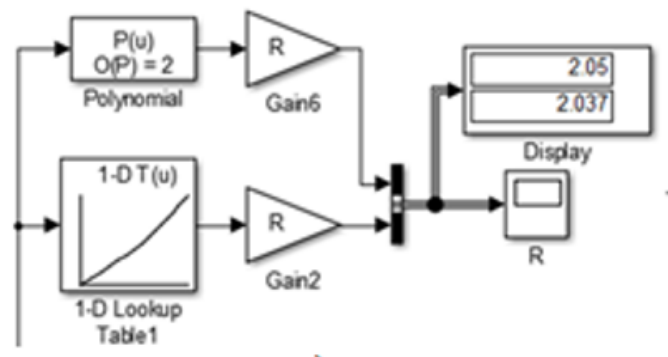

a)

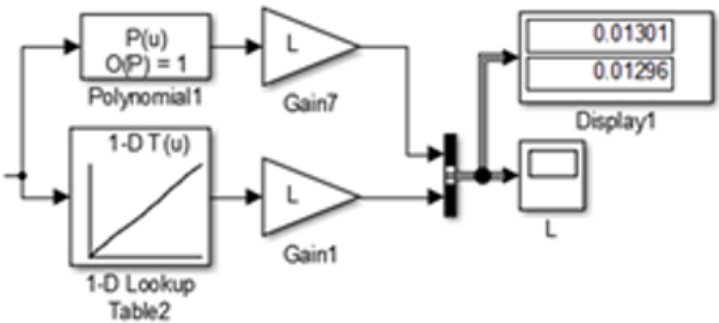

b)

Figure 5. Fragments of the Simulink-model of the analysis and monitoring of the active resistance and armature inductance: a) $R$; b) $L$.

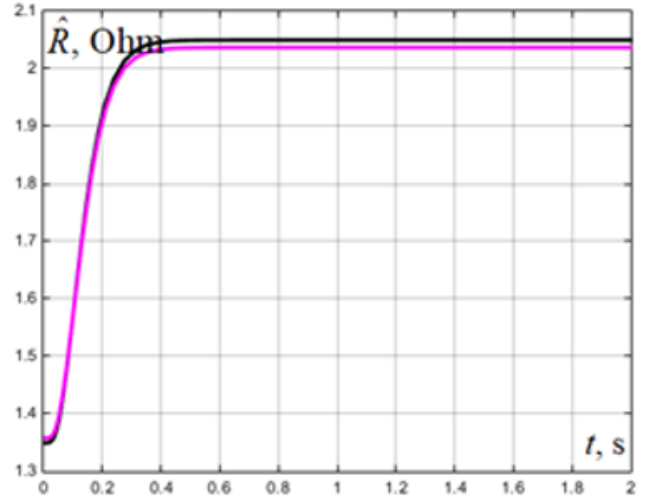

a)

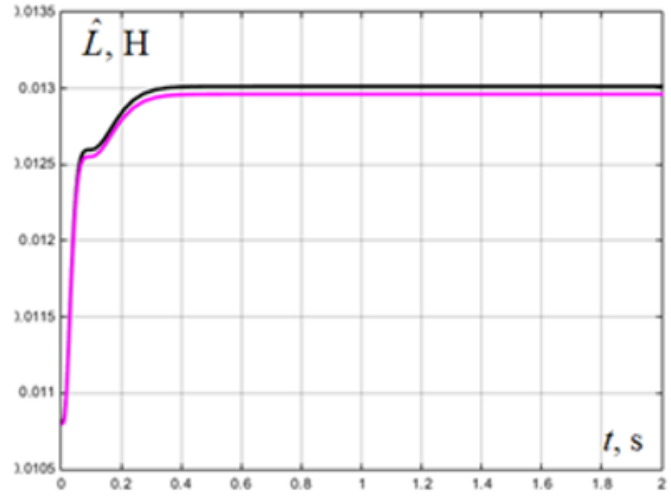

b)

Figure 6. Graphs of estimations: a) active resistance $R$; b) inductance $L$.

the analyzed parameters, respectively, $Q(R)$ and $Q(L)$.

On the basis of results yielded by computer simulation, functional dependencies of $f[Q(R)]$ and $f[Q(L)]$ were obtained, shown in figure 3 , which were approximated by the least squares method: $f[Q(R)]-$ quadratic function; $f[Q(L)]$ - linear function.

The dependencies shown in Figure 3 were implemented using 1-D Lookup Table blocks, and the respective functions using Polynomial blocks.

The final Simulink-model for analyzing and monitoring of the active resistance and armature inductance of a DC motor, based on intermediate calculations, is shown in Figure 4.

Fragments of the scheme of analyzing and monitoring the active resistance and armature inductance of the specified variations of the active resistance $R_{a \text {.var }}=1.5 R_{a}=2.037$ Ohm and inductance $L_{a \text {.var }}=1.2 L_{a}=0.013 \mathrm{H}$ are shown in figure 5 , and analyzed parameters evaluation schedule is shown in figure 6.

\section{Discussion}

The joint analysis of graphs and scheme fragments, reveals that the duration of the parameter estimation process does not exceed $0.4 \ldots 0.5 \mathrm{~s}$, and a maximum error (see figure 5) shall be $0.6 \%$ when evaluating the resistance using an approximating function and $0.3 \%$ when evaluating inductance using the functional dependence $f[Q(L)]$.

As a recent study has highlighted, this method provides the required high-accuracy characteristics at very high speed, which allows using the results to analyze and monitor objects with transient processes of short duration.

Thus, the method proposed can be applied for highprecision estimation of parametric disturbances in various electromechanical converters, both at the design stage and for the monitoring of the objects when in operation as a part of working sets.

\section{References}

[1] A.R. Gaiduk, K.V. Beskubova, A.S. Melnichenko, 2014 Research of dynamical systems stability at structural disturbances. (Taganrog: Proceedings of the Southern Federal University. Technical science, 5(154), 37-45.

[2] M. Zhixun, Z. Yuanzhe, S. Yan, L. Zhiming, L. Guobin., 2018 Constant switching frequency model predictive control for permanent magnet linear synchronous motor. (Transport systems and technologies, 4(3 S1), 279-288.

[3] Ivanovsky R.I., 2011 Applied aspects of the theory of sensitivity. (Scientific and technical statements of the St. Petersburg State Polytechnic University. 
Computer science. Telecommunications. Management, 6-1(138), 102-110.

[4] T.J. Abatzoglou, A fast maximum likelihood algorithm for frequency estimation of a sinusoid based on Newton's method (IEEE Trans. on Acoustics, Speech and Signal Processing, 33(1), (1985)

[5] D.H.T. Abramovitch, Henze D., 1998 An overview of the PES Pareto method for decomposing baseline noise sources in hard disk position error signals (IEEE Transactions on Magnetics, 34(1), 17-23.

[6] B.D.O. Anderson, 1998 From Youla-Kucera to identification, adaptive and nonlinear control 34(12), 1485-1506.

[7] B.D.O. Anderson, I.D. Landau, Least squares identification and the robust strict positive real property (IEEE Trans. on Circuits and Systems. 49(9), (1994)

[8] S. Bittanti, S.M. Savaresi, Safe estimate of sinusoidal signals for control applications (Phoenix: Proc. of the 38th Conf. On Decision \& Control) 28272832 (1999)

[9] M. Bodson, S. Douglas, Adaptive algorithm for the rejection of sinusoidal disturbances with unknown frequency. 33(12), 2213-2221 (1997)

[10] N.A. Malev, O.V. Pogoditsky, Statistical analysis of dynamic characteristics asynchronous electric motor with changing load parameters. (Kazan: Proceedings of the higher educational institutions. Energy sector problems; 21(1-2), 120-130 (2019)

[11] M.M. Katzman, Handbook of electric machines. (Moscow: Publishing Center "Academy") 480 (2005)

[12] V.I. Krupovich, Yu.G. Barybin, M.L. Samover, Handbook of power supply design. (Moscow: Energy) 456 (1980)

[13] V. Bavdekar, A. Mesbah, A polynomial chaos-based nonlinear Bayesian approach for estimating state and parameter probability distribution functions. (Boston: In Proceedings of the American Control Conference) 49-54 (2016)

[14] E.A. Buehler, J.A. Paulson, A. Mesbah, Lyapunov-based stochastic nonlinear model predictive control: Shaping the state probability distribution functions (Boston: In Proceedings of the American Control Conference) 258-269 (2016)

[15] M. Gevers, Identification for control: From the early achievements to the revival of experiment design (European Journal of Control, 11) 335-352 (2005)

[16] G. Guo, J. Zhang, Feedforward control for reducing disk-flutter-induced track misregistration (IEEE Transactions on Magnetics, 39(4), 2103-2108 (2003)

[17] F.R. Hansen, G.F. Franklin, R.L. Kosut, 1989 Closed-loop identification via the fractional representation: experiment design (Proc. Amer. Control Conf.) 386-391

[18] I.D. Landau, A stability theorem application to adaptive control IEEE Trans. on Aut. Contr., AC25(4), 814-817 (1980)

[19] A. Pantazi, J. Jelitto, N. Bui, E. Eleftheriou, Track-following in tape storage: Lateral tap emotion and control Mechatronics, 22(3), 361-367 (2012)
[20] B. Shahsavari, E. Keikha, F. Zhang, R. Horowitz, Adaptive repetitive control design with online secondary path modeling and application to bit-patterned media recording Magnetics, IEEE Transactionson, 51(4), $1-6(2015)$

[21] N.A. Malev, O.V. Pogoditsky, F.N. Lyubarchuk, Analysis of changes in the parameters of an asynchronous motor by a linear integral criterion using a reference model (Bulletin of KSPEU 1) 60-67 (2019) 